Ensino, Saúde e Ambiente-V5 (2), pp. 294-304, ago. 2012

\title{
TRILHAS INTERPRETATIVAS COMO INSTRUMENTO DE EDUCAÇÃO AMBIENTAL
}

\section{INTERPRETATIVE TRAILS AS A TOOL FOR ENVIRONMENTAL EDUCATION}

\author{
Vanusa Tubbs de Souza ${ }^{1}$, Fernando Augusto Santos Raggi ${ }^{2}$, Angela Soares dos Santos \\ Francelino $^{3}$, Ronaldo Figueiró ${ }^{4}$, Denise Celeste Godoy de Andrade Rodrigues ${ }^{5}$, Rosana \\ Aparecida Ravaglia Soares ${ }^{6}$ \\ 1,2,3,4,5,6 Centro Universitário de Volta Redonda - UNIFOA/MECSMA, \\ vanusaricobon@hotmail.com, fernando.agro@hotmail..com, angela.santos_19@hotmmail.com, \\ rravaglia@gmail.com \\ ${ }^{4}$ Centro Universitário Estadual da Zona Oeste - UEZO, ronaldofigueiro@gmail.com \\ ${ }^{5}$ Universidade do Estado do Rio de Janeiro - UERJ, Denise.cgar@ gmaill.com
}

\section{RESUMO}

O objeto do presente estudo é a interpretação de trilhas ecológicas em um Parque Natural, para implementação de práticas de ensino, através de estudos de campo. Uma Interpretação de trilhas se fundamenta na captação e tradução de informações referentes ao meio ambiente para quem a vivencia. A metodologia utilizada no presente estudo é uma análise quantitativa de estudos de casos do tipo descritivo. Foram realizadas excursões ao local, para determinação do trajeto, seguindo um plano de ação do ponto de vista teórico e prático, começando pelo levantamento dos recursos para subsídios dos eixos temáticos. Este estudo se justifica pela importância das trilhas ao estimular alunos acerca da preservação da natureza, o que deveria ser providencial em um país como o Brasil, onde há uma alta diversidade biológica e uma grande escassez de recursos. O objetivo do estudo é desenvolver uma aula prática para uma Educação Ambiental, abrangendo alunos de Graduação em Ciências Biológicas e Engenharia Ambiental

Palavras-Chaves: Parque Natural, Mata Atlântica, Biodiversidade, Ecologia.

\begin{abstract}
The object of the present study is the interpretation of nature trails in a natural Park, to implement teaching practices through field studies. An Interpretation of trails is based on capture and translation of information concerning the environment for people who experiences it. Case studies, descriptive, with a quantitative approach, are the methodology applied in the present study. Excursions to the site were taken in order to to determine the path, following a action plan in terms of theoretical and practical, starting with the survey of resources for grants of themes. This study is justified by the importance of trails to encourage students about the preservation of nature, which should be providential in a country like Brazil, where there is a high biological diversity and a severe shortage of resources. The objective is to develop a practical lesson for Environmental Education, involving undergraduate students in Biological Sciences and Environmental Engineering.
\end{abstract}

Key-Words: Natural Park, Atlantic Forest, Biodiversity, Ecology. 
Ensino, Saúde e Ambiente-V5 (2), pp. 294-304, ago. 2012

\section{INTRODUÇÃO}

A região de Volta Redonda apresenta uma elevada taxa de urbanização de seu território e são muito exíguas as chamadas áreas verdes disponíveis para o lazer, recreação e educação. A importância regional de fragmentos florestais como o Parque Natural Municipal Fazenda Santa Cecília do Ingá é grande, uma vez que o quadro de desmatamento na região do médio vale do Rio Paraíba do Sul assume valores muito elevados e são poucos os fragmentos de área superior a 100 ha. Alguns estudos foram empreendidos no perímetro do município como, por exemplo, (SOUZA et al., 2007), o que estudou um remanescente florestal de Mata Atlântica, na mesma região do estudo aqui proposto.

Devido a Mata Atlântica ser considerada a floresta tropical mais ameaçada do planeta, possuindo atualmente apenas $5,05 \%$ da sua cobertura original, o que pode levar rapidamente ao seu desaparecimento, faz-se necessário estudos visando uma vivência prática ser humano e natureza, buscando abordar temas que despertem uma conscientização em relação à preservação deste bioma. A Mata Atlântica constitui um dos biomas mais ricos em diversidade biológica do planeta e, por isso, considerado um dos cinco mais importantes hotspots de biodiversidade (JOLY et al. 1999).

No Estado do Rio de Janeiro estima-se que este bioma cobria originalmente em torno de $98 \%$ de seu território, considerando suas diferentes formações florestais principalmente a Floresta Ombrófila Densa, a Estacional Semidecidual e a Estacional Decidual, e ecossistemas associados, como manguezais, restingas e campos de altitude (Peixoto et al., 2004).

O objeto do presente estudo é a interpretação de trilhas ecológicas em um Parque Natural, para implementação de práticas de ensino, através de estudos de campo. Uma interpretação de trilhas ecológicas se fundamenta, na captação e tradução das informações referentes ao meio ambiente para quem vivencia. Contudo, um estudo de campo não lida apenas com a obtenção de informações, mas com significados, buscando firmar conhecimentos e despertar para outros novos, exercitar valores cognitivos, criar perspectivas, suscitar questionamentos fomentando a participação e trabalhando a percepção, a curiosidade e a criatividade humana.

Segundo Pádua (1997), a interpretação nas trilhas pode incluir atividades dinâmicas e participativas, em que o público recebe informações sobre recursos naturais, exploração racional, conservação, aspectos culturais, históricos, econômicos, arqueológicos e outros. As trilhas são guiadas e durante o percurso o monitor interpreta 
o ambiente utilizando as placas e o material de apoio, estimulando sempre a participação do grupo-alvo e despertando o interesse do mesmo. Assim, o grupo deixa de ser passivo para ser ativo "descobridor" do meio natural.

Como instrumento, a educação ambiental visa à integração socio-ambiental através do conhecimento dos recursos naturais e da valorização do meio ambiente, da transformação do ser humano em agente transformador e multiplicador das concepções obtidas e absorvidas e da melhoria da qualidade de vida. Um dos meios divulgados na interpretação ambiental é o dos percursos interpretativos, estes podem ser temáticos, com a predefinição de um tema antes da caminhada, ou de descoberta, ou turísticos e de lazer (TABANEZ \& PADUA, 1997).

As trilhas ecológicas interpretativas se enquadram dentro dos percursos interpretativos orientados metodologicamente, visam não somente a transmissão de conhecimentos, mas também propiciam atividades que revelam os significados e as características do ambiente por meio de experiência direta e por meios ilustrativos, sendo assim instrumento básico de programas de educação ao ar livre (POSSAS, 1999).

Acreditamos que as trilhas ecológicas constituem um instrumento pedagógico relevante, por nos permitir que em áreas naturais sejam criadas verdadeiras salas de aula ao ar livre e verdadeiros laboratórios vivos, suscitando o interesse, a curiosidade, a descoberta e possibilitando formas diferenciadas de aprendizado. As trilhas possibilitam também uma grande diversidade de eixos temáticos e abordagens ecológicas tanto com finalidades acadêmicas para utilização no ensino fundamental, médio e superior, bem como em atividades de pesquisa e investigação científica com finalidades de fornecer conhecimento e esclarecimento lúdico à comunidade em geral.

Diante deste contexto, as trilhas suscitam uma dinâmica de observação, de reflexão e de sensibilização, proporcionam uma diversificação de atividades e também um comportamento a ser adotado. Seu inconveniente é que exigem manutenção e fiscalização permanente, principalmente em áreas onde há ocupação urbana nas adjacências, como na área deste estudo.

Este estudo se justifica pela importância das trilhas ecológicas em estimular alunos e visitantes acerca da preservação da natureza, o que deveria ser providencial em um país como o Brasil, onde há uma alta diversidade biológica e uma grande escassez de recursos. Acreditamos que possa constituir um instrumento pedagógico prático e dinâmico, proporcionando uma aproximação à realidade dos temas abordados referentes à destruição da natureza, como a natureza reage às alterações causadas pelo homem, 
mas também, como a fauna e a flora ao interagir contribuem para a formação da biodiversidade.

Portanto, com o presente estudo pretendemos investigar e propor ações afirmativas que possibilitem a conservação da flora e fauna do Parque Natural Municipal Fazenda Santa Cecília do Ingá, localizado ao norte do município. Assim, o objetivo principal deste estudo é desenvolver uma aula prática para Educação Ambiental, visando abranger alunos do $1^{\circ}$ e $2^{\circ}$ período do curso de Ciências Biológicas e/ou graduandos do $1^{\circ}$ e $2^{\circ}$ período do Curso de Engenharia Ambiental, da Universidade de Volta Redonda - UNIFOA.

Pretendemos com este estudo, contribuir para a prática, ensino e pesquisa, para que docentes utilizem e se valiem deste estudo, buscando ferramentas para melhor aprimorar o ensino-aprendizado, levando a conscientização quanto à preservação da natureza. Outro de extrema relevância é contribuir com uma vivencia prática ser humano-natureza, para que o ensino de ciências venha não somente transmitir conhecimentos teóricos quanto aos conceitos de preservação, mas sim, contribuir para busca de valores ecológicos.

\section{METODOLOGIA UTILIZADA}

O estudo está sendo realizado em duas trilhas ecológicas no Parque Natural Municipal Fazenda Santa Cecília do Ingá, localizado no bairro Santa Cruz, na zona norte do município de Volta Redonda, Estado do Rio de Janeiro, sob as coordenadas latitude $22^{\circ} 27^{\prime} 34^{\prime}$ 'S e longitude 44 4' 51' 'W. Compreende 211 hectares e constitui-se no maior remanescente de Mata Atlântica do município (Figura1). 


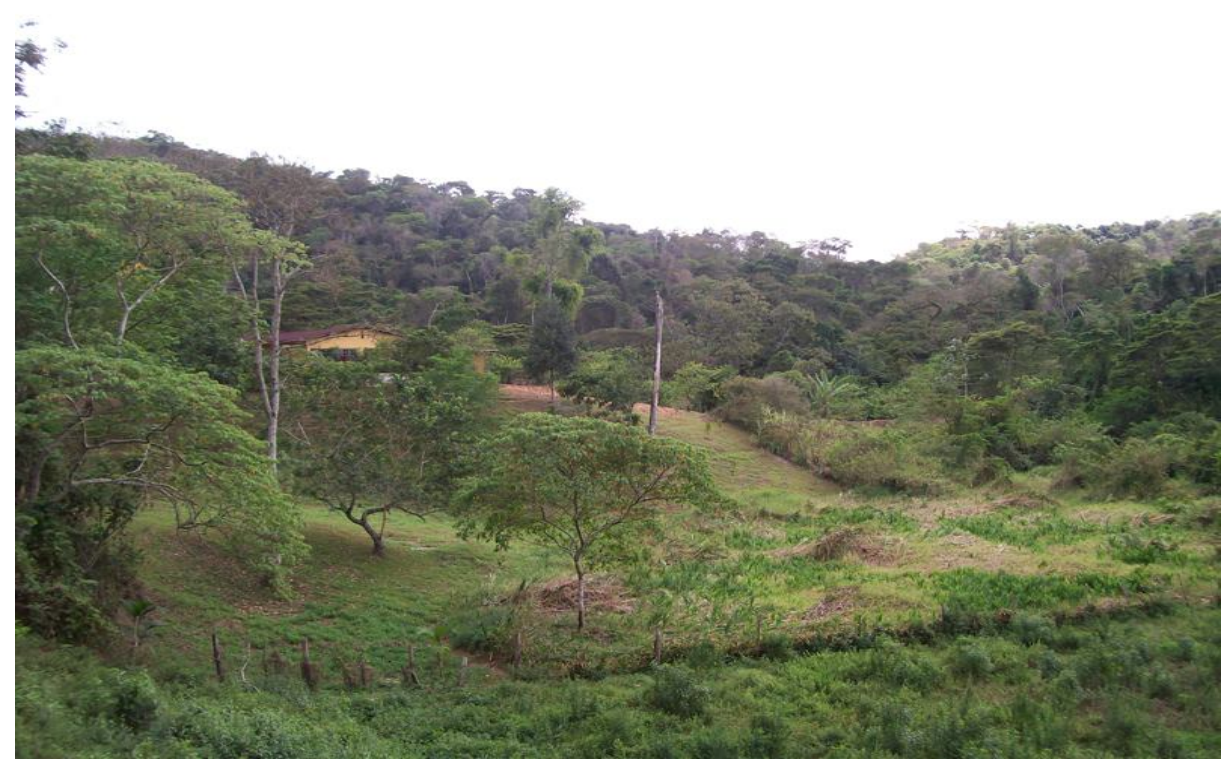

Figura 1: Foto do Parque Natural Municipal Fazenda Santa Cecília do Ingá, área deste estudo

A presente área de estudos foi escolhida devido à existência na literatura de um levantamento florístico prévio (SOUZA \& SOUZA, 2009), tendo publicado o artigo Composição Florística do Parque Natural Municipal Fazenda Santa Cecília do Ingá, Volta Redonda, RJ, Brasil, na Revista Sitientibus Série Ciências Biológicas, v. 9, n. 1, da Universidade Estadual da Feira de Santana - Bahia. O que possibilita um melhor desenvolvimento do projeto de educação ambiental proposto, bem como, possíveis temáticas como debates referentes às espécies de maior importância ecológica encontradas nas trilhas, e um melhor contato com o conhecimento local ou dos arredores, ampliando os estudos já realizados.

A natureza metodológica deste estudo é através de estudo de casos, onde se estuda determinado indivíduo e/ou grupos, examinando aspectos variados da vida. $\mathrm{O}$ estudo é do tipo descritivo, pois trabalha fatos cujos dados são colhidos da própria realidade e com uma forma de abordagem quali-quantitativa, traduzindo em números opiniões e informações para classificá-los e organizá-los, utilizando-se de métodos estatísticos.

O presente projeto tem como ponto de partida um acervo botânico com cerca de 129 espécies, já identificadas e herborizadas, sendo incorporadas ao acervo do herbário do Jardim Botânico do Rio de Janeiro, e suas duplicatas encontram-se no Centro de Monitoramento do Parque, para a montagem de coleção didática Para o inventário florístico, adotou-se o método de coletas aleatórias, privilegiando-se os componentes 
arbóreo-arbustivo, herbáceo e escandente.

O material foi coletado com tesoura de alta poda ou, quando necessário, através de escalada das árvores mais altas, adotando-se como critério de inclusão o diâmetro à altura do peito (tomado a $1,30 \mathrm{~cm}$ do solo) igual ou maior que $5 \mathrm{~cm}$ (DAP $\geq 5 \mathrm{~cm}$ ). Sendo anotadas, durante as atividades de campo, as características dendrométricas e biológicas dos exemplares amostrados. Sendo, portanto o ponto de partida para a elaboração de material didático-pedagógico e para subsidiar esse estudo de Educação Ambiental em trilhas interpretativas.

Para estruturar as trilhas interpretativas, foram feitas excursões de reconhecimento aos locais pretendidos, objetivando uma observação panorâmica dos locais quanto à acessibilidade e viabilidade dos mesmos, quanto à utilização de caminhos já abertos ou semi-abertos, partindo-se de um percurso pré-determinado com base no caminho feito pelos funcionários que utilizam esse trajeto para caminhar até a sede administrativa, ou seja, pela trilha principal do Parque. Posteriormente seguimos a definição da linha de atuação, com elaboração de um plano de ação do ponto de vista teórico e prático, começando pelo levantamento dos recursos para subsídios dos eixos temáticos: flora, fauna, ecologia, recursos hídricos e problemática ambiental (Figura 2).

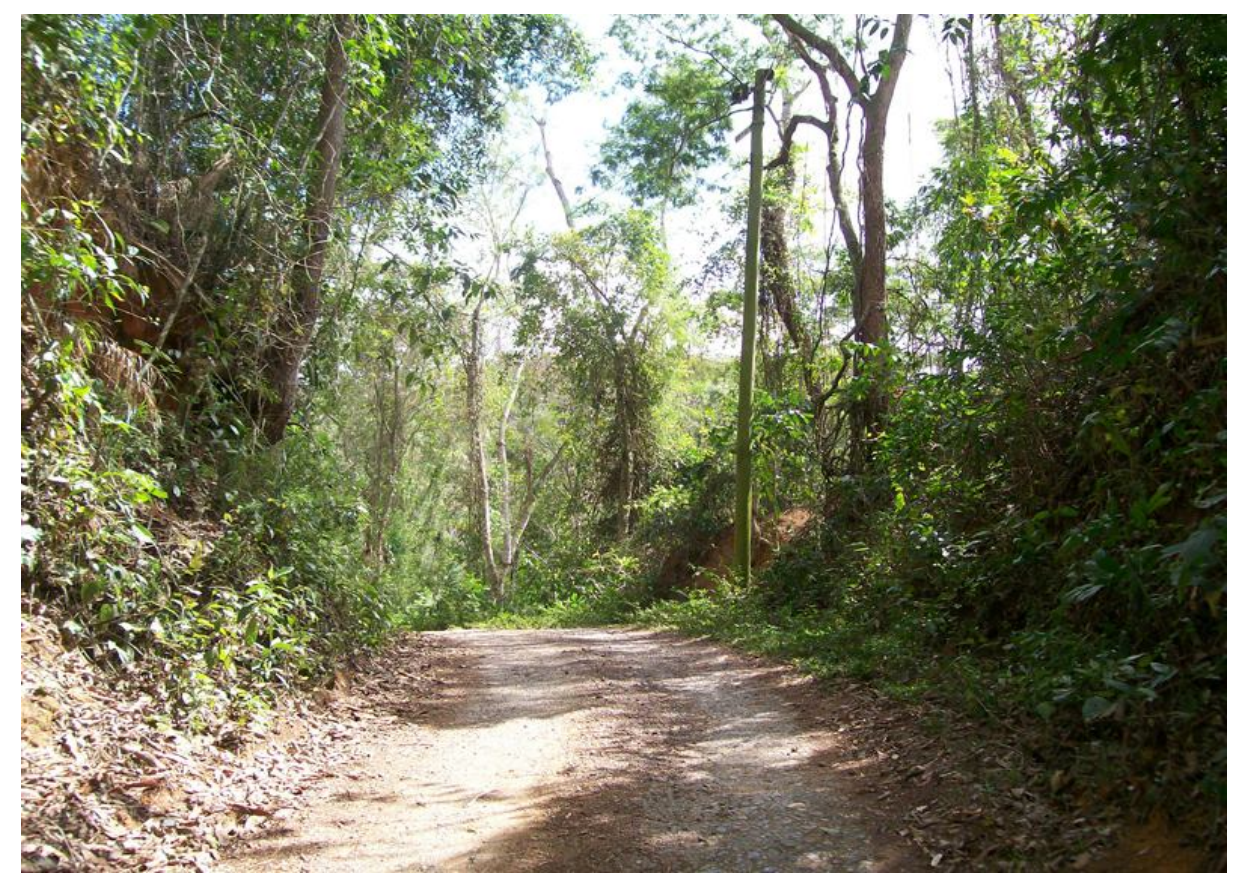

Figura 2: Foto da trilha principal do Parque

As espécies de maior importância ecológica já taxonomicamente classificadas nas famílias reconhecidas pelo “Angiosperm Phylogeny Group II" (APG, 2003) 
receberão placas interpretativas e educativas, feitas de material em PVC e fixadas ao solo. Após a produção, as placas serão encaminhadas ao laboratório de Biologia da Sede do Parque, para posterior colocação ao longo das margens da Trilha dos Pássaros e da Trilha das Borboletas, alternadamente em ambos os lados, identificando cada espécie selecionada, ver em Tabela 1.

As placas foram elaboradas utilizando-se do programa CorelDraw 12. Sendo adesivadas em material de PVC, com dimensões de $25 \mathrm{~cm}$ de comprimento, $18 \mathrm{~cm}$ de largura e 4,0 $\mathrm{mm}$ de espessura, com bordas arredondadas, fundo de cor verde transparência interativa. Para a descrição dos nomes vulgares; científicos e famílias, a fonte utilizada foi Arial Black, tamanho da fonte $\mathrm{n}^{\mathbf{o}} 38$, nas cores preto CMYK igual a: C (100); M (100); Y (100); K (100), e nas cores verde CMYK igual a: C (93); M (59); Y (90); K (40). Quanto ao nome ecologia, a fonte utilizada também foi Arial Black, tamanho da fonte $\mathrm{n}^{\circ} 26$, nas cores preto CMYK igual a: C (100); M (100); Y (100); K (100) (Figura 3).

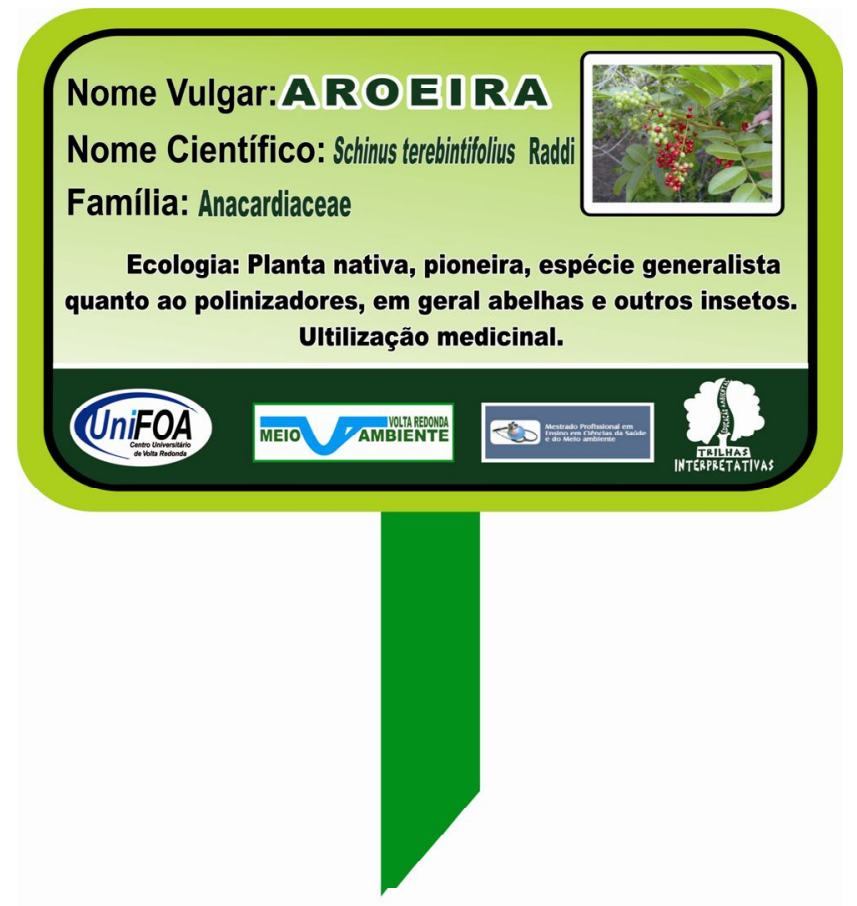

Figura 3: Placa Interpretativa, utilizada para identificação das espécies

Para elaboração do cabo das placas, foi utilizado material em PVC, pintados em cor verde, com dimensões de $50 \mathrm{~cm}$ de altura, $0,3 \mathrm{~cm}$ de largura e $0,4 \mathrm{~mm}$ de espessura. Estão sendo confeccionadas aproximadamente 70 placas, podendo sofrer alteração no numero de placas, devido ao estudo se encontrar em andamento. As mesmas serão 
colocadas em ambas as trilhas, para identificação das espécies selecionadas que farão parte deste projeto de Educação Ambiental.

Os alunos participantes do estudo receberão crachás de identificação. Para elaboração dos mesmos foi utilizado papel supremo 300g, na cor policromia (frente), tamanho $10 \times 15 \mathrm{~cm}$, perfurado com cordas de nylon. A logomarca utilizada é de criação da $1^{\mathrm{a}}$ autora do projeto (Figura 4 ).

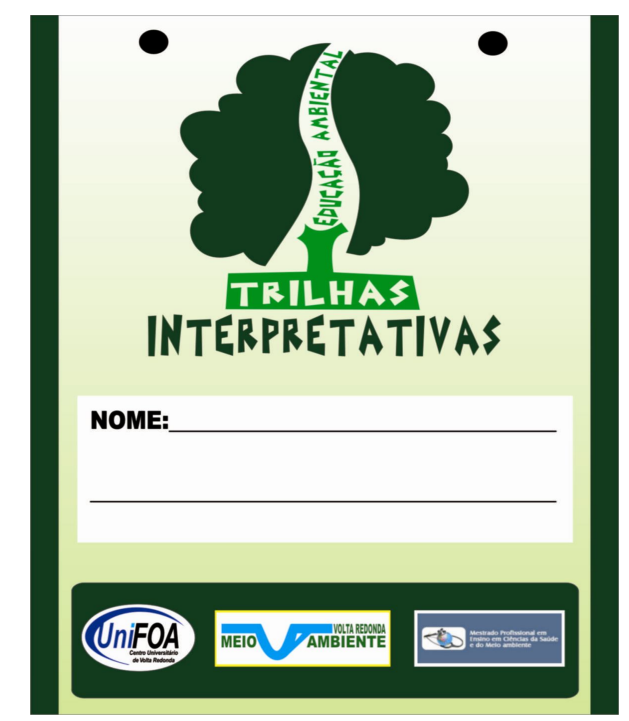

Figura 4: Crachá de identificação dos alunos participantes do estudo

Dentro do plano de atividades, foi elaborado um cronograma envolvendo: estudo bibliográfico, visitas às trilhas do Parque, demarcação das trilhas a serem percorridas, produção das placas indicativas, estudos de campo, colocação das placas, debates educativos, produção de materiais didáticos e elaboração de fichas de campo para coleta de informações sobre os atrativos da trilha. Para selecionar as espécies para o estudo, analisamos e obedecemos a critérios diferentes quanto à estruturação da trilha, infraestrutura física, atividades a serem desenvolvidas e principalmente sua importância ecológica. Portanto, esperamos com este estudo prover laboratórios naturais com fins educativos. 
Tabela 1. Lista das espécies de maior importância ecológica, selecionadas para o estudo de campo do Projeto de Educação Ambiental em Trilhas Interpretativas, amostradas no Parque Natural Municipal Fazenda Santa Cecília do Ingá, Volta Redonda, RJ.

\begin{tabular}{|c|c|c|}
\hline Família & Espécie & Nome vulgar \\
\hline Anacardiaceae & Schinus terebintifolius Raddi. & Aroeira \\
\hline Apocynaceae & Tabernaemontana fuchsiaefolia A.DC. & Leiteira \\
\hline Cannabaceae & Trema micrantha (L.) Blume & Pau-pólvora \\
\hline Euphorbiaceae & Euphorbia heterophylla (L.) & Leiteira \\
\hline Fabaceae & Caesalpinia echinata Lam. & Pau-Brasil \\
\hline Fabaceae & Caesalpinia ferrea Mart. & Pau-ferro \\
\hline Fabaceae & Caesalpinia peltophoroides Benth & Sibipiruna \\
\hline Fabaceae & Inga edulis Mart & Ingá \\
\hline Fabaceae & Inga uruguensis Mart & Ingá \\
\hline Fabaceae & Mimosa artemisiana Heringer \& Paula & Jurema-branca \\
\hline Fabaceae & Enterolobium contortisiliquиm (Vell.) & Orelha-de-macaco \\
\hline Fabaceae & Erythrina falcata Benth & Bico de papagaio \\
\hline Fabaceae & Piptadenia gonoacantha Mart. & Pau- jacaré \\
\hline Fabaceae & Schizolobium parahyba (Vell) & guapuruvu \\
\hline Lauraceae & Nectandra menbranaceae (S.w.) Griseb. & Canela - preta \\
\hline Lauraceae & Nectandra opositifolia Nees & Canela - ferrugem \\
\hline Malvaceae & Chorisia speciosa St. Hil. & Paineira \\
\hline Myrtaceae & Eugenia uniflora L. & Pitanga \\
\hline Myrtaceae & Psidium guajava $\mathrm{L}$. & Goiabeira \\
\hline Myrtaceae & Psidium catteyanum & Araça \\
\hline Sapindaceae & Cupania oblongifolia Mart. & Camboatã \\
\hline Salicaceae & Casearia sylvestris (SW) & Guaçatonga \\
\hline Siparunaceae & Siparuna guianensis - Aubl. & Siparuna \\
\hline Urticaceae & Cecropia glaziovi Snethlage & Embaúba \\
\hline
\end{tabular}

\section{CONCLUSÕES}

Com as aulas práticas de Educação Ambiental realizadas no Parque, através da interpretação das trilhas, utilizando-se de espécies selecionadas, esperamos contribuir 
com o ensino-aprendizado dos alunos participantes. E também, para que este estudo sirva como instrumento metodológico, onde o ensino de ciências venha não somente transmitir conhecimentos teóricos quanto aos conceitos de preservação da natureza, mas sim venha transmitir através das aulas práticas de campo, a importância ecológica das espécies existentes na área de estudo. Espera-se também contribuir para busca valores ecológicos, buscando uma avaliação e observação, fazendo com que este instrumento utilizado, não somente seja relevante para a conscientização quanto à conservação da flora e da fauna do Parque, como também para a preservação de outros fragmentos florestais de Mata Atlântica em nossa região.

\section{REFERÊNCIAS BIBLIOGRÁFICAS}

APG. 2003. An update of the Angiosperm Phylogeny classification for the orders and families of flowering plants: APG II. Botanical Journal of the Linnean Society 141: $399-436$.

JOLY, C.A., AIDAR, M.P.M., KLINK, C.A., MCGRATH, D.G., MOREIRA, A.G., MOUTINHO, P., NEPSTAD, D.C., OLIVEIRA, A.A., POTT, A., RODAL, M.J.N. \& SAMPAIO, E.V.S.B. 1999. Evolution of the Brazilian phytogeography classification systems: implications for biodiversity conservation. Ciência e Cultura 51:331-348.

PADUA, S.M. 1997. Cerrado Casa Nossa: um projeto de educação ambiental do jardim botânico de Brasí lia. Brasília. UNICEF. 35pp.

POSSAS, I. M. 1999. Programa GUNMA: Integrando Parque Ecológico e Comunidade no município de Santa Bárbara do Pará. Dissertação de Mestrado. Universidade Federal do Pará. 73pp.

PEIXOTO, G.L; MARTINS, S.V; SILVA, A.F \& SILVA, E. 2004. Composição Florística do Componente arbóreo de um trecho de Floresta Atlântica na área de proteção Ambiental da serra da Caipora Grande, Rio de Janeiro, RJ, Brasil. Acta Bot.Bras. 18 (1): Jn/Mar.

SOUZA, G. R; PEIXOTO, A.L; FARIA, M.J.B \& ZAÚ, A.S. 2007. Composição 
Ensino, Saúde e Ambiente -VV5 (2), pp. 294-304, ago. 2012

florística e aspectos estruturais do estrato arbustivo-arbóreo de um trecho de floresta Atlântica no médio vale do Rio Paraíba do Sul, Rio de Janeiro, Brasil. Sitientibus Série Ciências Biológicas 7(4): 398-409.

TABANEZ, M. F. \& PADUA, S.M. (orgs.) 1997. Educação Ambiental: caminhos trilhados no Brasil. Instituto de Pesquisas Ecol ógicas - IPÊ. Brasília. 283 pp. 